\title{
The emerging roles of mannose-binding lectin-associated serine proteases (MASPs) in the lectin pathway of complement and beyond
}

\author{
József Dobó ${ }^{1}$, Gábor Pál$^{2}$, László Cervenak ${ }^{3}$, Péter Gál ${ }^{1}$ \\ ${ }^{1}$ Institute of Enzymology, Research Centre for Natural Sciences, Hungarian Academy of Sciences, \\ Budapest, Hungary \\ ${ }^{2}$ Department of Biochemistry, Eötvös Loránd University, Budapest, Hungary \\ ${ }^{3}$ 3rd Department of Internal Medicine, Semmelweis University, Budapest, Hungary
}

Correspondence to:

Peter Gál

Institute of Enzymology

Research Centre for Natural Sciences

Hungarian Academy of Sciences

Magyar tudósok körútja 2

$\mathrm{H}-1117$ Budapest, Hungary

Tel.: +36-1-3826768, Fax: +36-1-3826295

e-mail: gal.peter@ttk.mta.hu, 
Running title: MASPs in the lectin pathway and beyond

Summary: Mannose-binding-lectin (MBL)-associated serine proteases (MASPs) are the enzymatic constituents of the lectin pathway of the complement system. They are complexed with large pattern recognition molecules (PRMs) such as $\mathrm{MBL}$, other collectins, and ficolins. The main function of two out of the three MASPs has crystallized lately: MASP-1 autoactivates first, then it activates MASP-2, and finally both participate in the formation of the C4b2a convertase. In addition to this both enzymes are involved in several other processes, which are subject to intense research nowadays. Notably MASP-1, as a promiscuous enzyme, has been implicated in the coagulation cascade, in the kinin-generating contact system, and in cellular activation through protease-activated receptor (PAR) cleavage on endothelial cells. The third protease MASP-3 has emerged recently as the protease responsible for pro-factor $D$ activation in resting blood, providing a fundamental link between two complement pathways. At present all three MASPs have at least one well-defined role and several other possible functions were implicated. Defect or more likely over-activation of MASPs may culminate into diseases such as ischemia-reperfusion injury (IRI) hence MASPs are all potential targets of drug development.

Keywords: complement, lectin pathway, alternative pathway, MBL-associated serine protease, coagulation, protease-activated receptor (PAR) 


\section{Introduction and brief history}

When Ikeda and colleagues showed in 1987 that a blood-borne lectin isolated from rat serum (MBP which is now called MBL) is capable of hemolysing sheep erythrocytes coated with yeast mannan (1), a new era of research began that changed our view about the complement system for ever. First, it was assumed that MBL (mannose-binding lectin, aka mannan-binding lectin, or mannan-binding protein) only supplements the well known classical pathway, since it can bind and activate the tetramer consisting of two $\mathrm{C} 1 \mathrm{r}$ and two $\mathrm{C} 1$ s proteases (2). Later, it became gradually evident that a new, independent activation pathway of the complement cascade had been discovered. The scientific impact of this discovery can be compared only to the discovery of the alternative (properdin) pathway by Pillemer in the early 1950s (3). The lectin pathway, the youngest according to the chronology of discovery, represents a very ancient type of innate immune defense. As an ancient pathway, the lectin pathway is intimately connected to the classical and the alternative pathways. In this review we will delineate the mechanism of lectin pathway activation, the cross-talk between the lectin pathway and the other complement pathways and we will also give account of the role of the lectin pathway enzymes in the innate immune defense outside the complement system.

Genome encoded pattern recognitions molecules ("receptors") are present everywhere in the body: inside the cells, on the cell surface and in the extracellular space. In the bloodstream, pattern recognition molecules circulate to monitor the presence of invading pathogens and dangerous self-structures (e.g. dead cells, cancer cells). C1q, the pattern recognition molecule of the classical pathway binds mainly to immune complexes, bridging the innate and adaptive immune responses. MBL and the other pattern recognition molecules of the lectin pathway recognize the dangerous structures immediately as they appear in the bloodstream long before the development of the adaptive immune response. The lectin pathway therefore represents the very first line of defense against infection. Since the discovery of the $\mathrm{MBL}$ the inventory of the pattern recognition molecules has been continuously increasing (4). MBL, ficolin-1,-2,-3, collectin10 (CL-L1), collectin-11 (CL-K1) are the known molecules that can initiate lectin pathway activation. After the pattern recognition molecules bind to the dangerous particles an enzymatic signal is generated by associated serine proteases. These serine proteases are present as zymogens in the circulating initiation complexes and become activated upon the pattern recognition molecules bind to their targets. The first enzymatic step is usually an autoactivation. The initial proteolytic signal is then further amplified by a proteolytic cascade. There are three MBL-associated serine proteases (MASPs), MASP-1, -2 and -3 . Since MASP-1 is the most abundant one (having 140 nM serum concentration), it was discovered first and it was believed that it is a C1s-like protease, as the MASP-1 preparation cleaved C4 and C2 generating the classical/lectin pathway C3 convertase complex (C4b2a) (5). Later it turned out that the C1s-like protease responsible solely for C4 and partially for C2 cleavage was in fact a minor "contaminating" component co-purified originally with MASP-1, it was the later discovered MASP-2 (having 6 nM serum concentration) (6). MASP-1 
can autoactivate and cleave C2 but not C4, therefore cannot generate C3 convertase (7). It was also shown that MASP-2 can autoactivate in vitro and an initiation complex consisting of MBL and MASP-2 molecules can initiate lectin pathway activation without the contribution of any other proteases (8). This view about the superiority of MASP-2 persisted for more than a decade while the physiological role of MASP-1 was a subject of intense debate among complementologists. In the meantime in 2001, quite unexpectedly, a third MBL-associated protease (MASP-3) was discovered (9). The physiological role of this protease was even more mysterious than that of MASP-1: in spite that MASP-3 binds to the pattern recognition molecules, it cannot cleave any components of the $\mathrm{C} 3 / \mathrm{C} 5$ convertase complexes. Consequently, when adding MASP- 3 to the MBLMASPs complexes it acts as an inhibitor of lectin pathway activation as it displaces MASP-2 and MASP-1. Moreover, C1 inhibitor, the natural serpin type inhibitor of C1r, C1s, MASP-1 and MASP-2 does not inhibit the activity of MASP-3 on synthetic substrates (10). Besides the three serine proteases, two non-catalytic proteins can also be found in the initiation complexes. MAp19 (alias MAP-2 or SMAP) consists of the two N-terminal domains (CUB1 and EGF) of MASP-2 (11). MAp44 (alias MAP-1) represents the four N-terminal domains of MASP-1 (CUB1, EGF, CUB2 and CCP1) (12). Now we have two genes and five gene products due to alternative spicing (Fig. 1). MASP-1, MASP-3 and MAp44 are the alternative splice products of the MASP1 gene, whereas MASP-2 and MAp19 are that of the MASP2 gene.

The picture of lectin pathway activation is quite complicated since we have at least six pattern recognition molecules, three associated serine proteases and two associated proteins. The composition and the stoichiometry of the initiation complexes are not well characterized and there are many open questions regarding the role of the individual components in the complex mechanism of the initiation of the innate immune response. The significance of the research of lectin pathway is underlined by the fact that uncontrolled activation of this pathway contributes to the development and worsening of serious disease conditions (e.g. myocardial infarction, stroke) in which the proteolytic activity of MASPs plays a major role (13). During the recent years there has been an intense progress in the characterization of enzymatic and structural properties as well as the physiological roles of the MBL-associated serine proteases. In the following chapters we will provide an up-to-date picture about the actions of MASPs in the complement system and beyond.

\section{The individual roles of MASPs in lectin pathway activation}

\section{General approaches to study protein networks}

Like most biological processes, the activation of the lectin pathway is also governed by specific protein-protein interactions and some of these interactions also involve highly specific proteolytic steps. With other words, the lectin pathway is a protein network and also a proteolytic cascade. There are three major approaches to study the mechanism of action of protein networks. 
Nowadays a quite widely used approach is to genetically remove individual components from the in vivo system either by gene knockout (KO) or gene silencing (gene knockdown) experiments. Such powerful experiments can often quickly identify major functions of the individual proteins. However, several important aspects of $\mathrm{KO}$ experiments should be carefully considered when interpreting the results. When KO animals or genetically deficient humans are studied one should be aware that the corresponding protein has been missing throughout the entire embryogenesis and in fact the entire lifetime of the individual. This might generate complex indirect effects including compensatory biological processes. In addition, in a network, individual components might have multiple interacting surfaces for multiple network components. Removal of such central components can disrupt many interactions simultaneously and the observed results can be again very complex.

When applying the classic biochemical approach, which is also the most tedious one, each member is isolated from its natural source or produced as recombinant protein. Then, each protein is individually characterized in vitro both functionally (binding characteristics, stability, enzymatic properties etc.) and structurally (X-ray crystallography and / or NMR). Then, the well characterized purified compounds are combined and further analyzed in various complexes to ultimately reconstruct the original network. If all components have indeed been identified, ideally, the reconstructed network should recapitulate the functional properties of the natural one.

The third approach is to keep the original network as close to the natural state as possible and disrupt individual protein interaction sites questioning the function of a single protein-protein interaction or a single enzyme activity. To do so, one needs to have a natural or manmade compound that selectively binds to the given binding site with sufficiently high affinity. We decided to decipher individual roles of each MASP enzyme in lectin pathway activation by combining the latter two approaches.

\section{Development of specific MASP-1 and MASP-2 inhibitors}

As an important first step we established a robust bacterial expression system to produce the catalytic fragments of the MASP enzymes in large quantities and high quality. These fragments do not dimerize or bind to pattern recognition molecules, but carry all the enzymatic properties of the entire protease. Zymogens of all three MASP enzymes were expressed in $E$. coli leading to nontoxic inclusion bodies and the proteins were taken through a refolding procedure to generate correctly folded native forms (7).

Even this isolation procedure was quite informative. Whatever we tried, zymogen MASP-1 went through autoactivation at early phase of the isolation process suggesting high autoactivation potency. On the other hand, zymogen MASP-2 became activated only during a later chromatographic step which caused high local concentration of the proenzyme. We were able to prevent the process by keeping the concentration low and performing the purification at low temperature (14). Zymogen MASP-3 on the other hand did not autoactivate at all. 
It was shown by Vorup-Jensen et al (8) that at low concentration, or when complexed with MBL in solution, proenzyme MASP-2 remained in zymogen form. On the other hand, when the MBL-zymogen MASP-2 complex was transferred onto mannan-coated surface, MASP-2 autoactivation occurred. Nevertheless, the authors also noted that the rate of MASP-2 activation was considerably lower than observed in natural MBL-MASP complexes generated by incubating $\mathrm{MBL}$ with MBL-deficient serum. These latter complexes have a more diverse composition containing not only MASP-2, but also MASP-1 and other MBL associated components.

It has been gradually established by the contribution of several research groups that MASP3 cannot cleave either C2 or C4; MASP-1 is unable to cleave C4 but cleaves C2; and among the MASPs only MASP-2 can cleave both C2 and C4 generating a C4b2a type C3 convertase $(6,7,9,15$ 18).

This knowledge combined with the observed characteristic autocatalytic properties of the three MASP enzymes led to a perception and an activation model that dominated the field for over a decade. Namely, MASP-2 was considered as the single key initiator of the lectin pathway activation as it can autoactivate and generate a C3 convertase by its own. MASP-1 was considered to be at most an auxiliary enzyme that provides some of the activated C2 component for the convertase (19), although it was also shown in a murine model that MASP-1 facilitates MASP-2 activation through an unknown mechanism (20). MASP-3 was believed to be a negative pathway regulator.

This was the state of knowledge when we decided to develop MASP-1 and MASP-2 inhibitors for two different purposes. Accepting the view that MASP-2 is the single key enzyme we aimed to develop a specific MASP-2 inhibitor to selectively block the lectin pathway while leaving the other two pathways fully functional. This was important as such inhibitors have been unknown from nature. The major negative regulator of the lectin pathway, the $\mathrm{C} 1$ inhibitor serpin is also a potent inhibitor of the classical complement pathway enzymes. We also aimed to develop a specific inhibitor against MASP-1 in order to quantitatively assess its contribution to the lectin pathway.

By that time we had already solved the X-ray structures of both the zymogen and the active form of MASP-2 and also the active form of MASP-1 $(21,14,22)$. Based on the active enzyme structures it was clear that compared to the archetype proteinase trypsin, the substrate binding cleft of MASP-1 is in some degree while that of MASP-2 is largely blocked by large surface loops. This suggested that our novel inhibitor should be as small as possible in order to minimize the degree of clash with the large enzyme loops.

But how can one develop a selective binder against a particular protein site? To our knowledge in silico methods are not powerful enough yet, and the only experimental approaches are either selection of the binders from a library of synthetic small molecules or developing a protein binder through directed evolution.

Small molecules (below 500 Dalton) can be very useful but are usually limited to cases when the target has a well-defined deep binding pocket and that pocket is unique to the target 
protein i.e. there are no other related proteins with identical or highly similar binding sites. This is clearly not the case with the MASP enzymes. These enzymes are all trypsin-like enzymes having a deep S1 pocket (23) with a negatively charges Asp at its bottom. The plasma contains dozens of evolutionary related trypsin-like serine proteinases, e.g. the members of the blood coagulation cascade, the three complement activation pathways and the complement convertases, and also plasminogen activator, plasmin, kallikreins etc. There are many known small molecule serine proteinase inhibitors, but almost all of them are quite nonspecific inhibiting multiple trypsin-like enzymes with similar efficiency.

So, we decided to develop MASP-1 and MASP-2 inhibitors by evolving them from an existing natural substrate-like reversible trypsin inhibitor. To minimize the clash with the substrate binding cleft covering enzyme loops our first choice was the 14 residue Sunflower Trypsin Inhibitor (SFTI), which is the smallest natural serine proteinase inhibiting peptide. Nevertheless, it is still much larger than a typical small molecule promising considerable specificity.

We prepared a library by randomizing six presumed proteinase contacting positions of SFTI through combinatorial mutagenesis. The SFTI-library was displayed on the surface of M13 phage in a monovalent fashion and selected for binding separately on MASP-1 and MASP-2 (24). After the phage display selection procedure we chose two clones that as a set provided the best research tools. One of these, SFMI-2 inhibited MASP-2 with $180 \mathrm{nM}$ equilibrium inhibition constant $\left(\mathrm{K}_{\mathrm{l}}\right)$ while did not inhibit MASP-1 at all, while the other one, SFMI-1 had a $65 \mathrm{nM} \mathrm{K}$ on MASP-1 but also inhibited MASP-2 with a micromolar $K_{1}$ value.

In the WIESLAB Complement system Screen assay (25), which measures the activation of the three complement pathways separately, SFMI-2 proved to be a selective lectin pathway inhibitor, the very first of its kind, with a $9.9 \mu \mathrm{M} I \mathrm{C}_{50}$ value. The observed complete pathway inhibition was in perfect accordance with the generally accepted central role of MASP-2. SFMI-1 turned to be also a selective lectin pathway inhibitor having a $3.2 \mu \mathrm{M} \mathrm{IC}_{50}$ value. Based on the prevailing activation model of the lectin pathway the potency of this inhibitor was unexpectedly high. Compared to SFMI-2 SFMI-1 is a 6-fold weaker MASP-2 inhibitor, but it is actually a 3-fold more efficient lectin pathway blocker, which altogether represents a 20-fold discrepancy.

To dissect the potential source of this discrepancy we conducted a C3 and a C4 deposition assay, both designed such that the inhibitor was added to non-activated serum containing the PRM-associated MASPs in proenzyme form and then the mixture was transferred to mannancoated activating surface. Both assays recapitulated the WIESLAB assay results: SFMI-1, which inhibits both MASP-1 and MASP-2 was 15-20-fold more potent than SFMI-2. We also performed a different type of C4-deposition assay, in which we first activated the MBL-bound MASPs on mannan-coated wells and then added purified C4 mixed with either SFMI-1 or SFMI-2 and measured $\mathrm{C} 4 \mathrm{~b}$ deposition (26). In these assays the ratios of $\mathrm{IC}_{50}$ values of the two inhibitors did not track with those in the WIESLAB assay and the previous C3 and C4 deposition assays, but these ratios were in good accordance with the $K_{1}$ values of SFMI-1 and SFMI-2 measured on MASP-2. We concluded that once MASP- 2 is already activated, the MASP-1 inhibiting capacity of SFMI-1 has no 
relevance and only its MASP-2 inhibiting capacity matters. On the other hand, when we started with proenzyme MASPs, inhibiting MASP-1 had a major contribution to the suppression of MASP-2 function. Altogether the results proved that MASP-1 contributes to MASP-2 activation, but the quantitative proportion of this contribution could not have been determined without a MASP-1 selective inhibitor.

In order to solve this problem we decided to evolve a second generation of MASP inhibitors starting with a member of a different substrate-like inhibitor, namely the Pacifastin family. We did so, because our results with the SFMI inhibitors suggested that their small size, which we assumed to be important for avoiding steric clash, might actually limit their selectivity. The Pacifastin inhibitor SGPI-2 that we chose is a 3-disulfide stabilized 35 amino acid residues small protein that provides a larger interacting site for the enzymes. After phage display selection of an SGPI library we tested the inhibitory characteristics of several variants produced as recombinant proteins. We decided to use two variants for further studies. One of them, SGMI-1 is a 7 nanomolar inhibitor of MASP-1 and a 58 micromolar inhibitor of MASP-2, while the other one, SGMI-2 is a 6 nanomolar inhibitor of MASP-2 and it does not inhibit MASP-1 at all (27). With this monospecific pair of inhibitors we could finally analyze the selective contribution of MASP-1 to the activation of MASP2 and the lectin pathway in general.

SGMI-2, as expected was found to be a potent lectin pathway inhibitor. Compared to SFMI2 SGMI-2 binds 30-fold tighter to MASP-2 (6 nM versus $180 \mathrm{nM} \mathrm{K}$ value on MASP-2) and based on the $I_{50}$ values of the WIESLAB assays SGMI-2 is 150-times more potent lectin pathway blocker than SFMI-2 (66 nM versus $9.9 \mu \mathrm{M}$ value). Importantly, SGMI-2 is also pathway selective as it does not inhibit the classical or the alternative pathways and it is ineffective on the C3 and C5convertases as well.

\section{MASP-1 is the exclusive activator of MASP-2}

When testing SGMI-1 in serum-based lectin pathway activation assays we expected partial inhibition in accordance with the presumed contributing role of MASP-1 to MASP-2 activation. In contrast, to our great surprise, SGMI-1 completely inhibited lectin pathway activation (28). Moreover, SGMI-1 turned to be more potent than SGMI-2 in all assays that started with the PRMassociated MASPs being in their zymogen form. To this end we conducted many assays. The assays differed in serum dilution (we even performed a whole blood assay); we tested MBL- versus ficolin-based activation; C3b, C4b deposition or MAC generation. Similarly to SGMI-2, SGMI-1 is also pathway selective as it does not inhibit the classical or the alternative pathways or any of the C3 and C5-convertases.

Along the same logic we followed in the case of the SFMI inhibitors, we performed an important control experiment, in which we pre-activated the serum either on mannan-coated or on acetylated BSA-coated surface to trigger activation of MBL-associated and ficolin-associated 
MASPs, respectively, and added isolated intact C4 mixed either with SGMI-1 or SGMI-2. In this arrangement only SGMI-2 could inhibit C4b deposition, SGMI-1 was totally inert.

In all, we concluded that in normal human blood MASP-1 is the exclusive activator of MASP-2. This means that even though many research groups including ours detected that MASP-2 can autoactivate in vitro, this capacity of the enzyme simply does not manifest in vivo (28). Therefore the previously accepted text book mechanism of lectin pathway activation was incorrect. Based on our mono-selective pair of inhibitors we could detect the error and correct it (Fig. 2). Soon after, an independent study fully corroborated our findings: Degn et al (29) reported the lack of functional lectin pathway in a human patient genetically deficient in MASP-1 (and MASP-3) and they also showed that adding exogenous MASP-1 to the serum of the patient restored the lectin pathway.

Our monospecific inhibitors also allowed us to answer a more subtle but nevertheless very important and long-lasting question: what are the relative contributions of MASP-1 and MASP-2 to generating the cleaved $\mathrm{C} 2$ component of the C4b2a C3 convertase? We determined this through measuring C3 deposition on C4b-saturated surfaces using SGMI-1 and SGMI-2. When MASP-1 was inhibited by SGMI-1, the level of C3 deposition dropped to $43.5 \%$ of the non-inhibited experiment. When MASP-2 was inhibited by SGMI-2, the level of C3 deposition dropped to $61 \%$ of the noninhibited value. The two independent measurements unequivocally showed that MASP-1 is the major contributor of the C2 cleavage. When applying both inhibitors at the same time the level of C3 deposition was reduced to $3.3 \%$.

The fact that the inherent auto-activating capacity of MASP-2 observed in various in vitro assays from several research groups did not manifest in normal human blood inspired us to quantitatively determine the auto-activation and cross-activation capabilities of MASP-2 and MASP-1 (30).

\section{Zymogen MASP-1 exerts the first proteolytic steps}

Auto-activation is a two-step process. It starts with a proenzyme molecule activating another proenzyme molecule of the same protease. We introduced the term for this step: zymogen autoactivation. The next step is when the already activated proteinase activates a zymogen molecule of its own type and we call that autocatalytic activation. Both activation types have potential cross-activation versions where the two enzymes are different and we call these as zymogen-zymogen cross-activation and active-zymogen cross-activation.

In the context of two enzymes (in this case MASP-1 and MASP-2) there are two zymogen autoactivation reactions, two autocatalytic activation reactions, two zymogen-zymogen crossactivation reactions and two active-zymogen cross-activation reactions, altogether 8 pair-wise enzyme-substrate reactions to analyze.

In such assays a zymogen molecule is both a (usually low-activity) enzyme as well as a substrate. As the reaction proceeds, the concentration of the zymogen gradually decreases while 
that of the activated form increases. Moreover, as catalytic efficiency of the zymogen and the active form are usually several orders of magnitude different, it is a challenge to de-convolute individual catalytic constants from such reactions.

In order to make the system manageable we separated the substrate and enzyme roles of the zymogens into two mutant molecules. By producing a zymogen in which the catalytic triad serine is replaced with an alanine we generated a form that can act only as a substrate, and even when it is processed at the activation site, the cleavage results in an inactive species. In the other type of mutants we replaced the activation cleavage site Arg with a Gln rendering this site uncleavable by trypsin-like proteinases. Such mutant proenzymes cannot function as substrates but can fulfill the enzymatic role of the (low activity) proenzyme.

We aimed to compare the relative inherent capabilities of the MASP (pro)enzymes to perform these auto- and cross-activation reactions and therefore we placed the project out of the context of the PRMs using recombinant catalytic fragments of the MASPs. Cleavages were followed as a function of time on SDS PAGE, the gels were analyzed by densitometry, and individual rates as well as $\mathrm{k}_{\mathrm{obs}} /[\mathrm{E}]_{\mathrm{T}}\left(\sim \mathrm{k}_{\mathrm{cat}} / \mathrm{K}_{\mathrm{M}}\right)$ values were calculated. The 8 pair-wise results are summarized in Fig. 3 (30).

The study led to the following major conclusions. Proenzyme MASP-2 has a hardly measurable zymogen autoactivation capability. Previous experiments detected zymogen MASP-2 autoactivation only at very high local concentration of the proenzyme either on an ion-exchange chromatography column or in the context of MBL, saturated with the proenzyme. As we already proved that in vivo only MASP-1 activates proenzyme MASP-2, it follows that this kind of high local concentration of proenzyme MASP-2 is not achieved in vivo. Note that proenzyme MASP-2 has a low plasma concentration representing only a minor component of the MBL (and ficolin)associated proteins $(31,32)$.

On the other hand the quantitative study clearly showed that once MASP-2 is activated it becomes a potent activator of both proenzyme MASP-2 and pro-enzyme MASP-1. Based on the results of our (and others) in vitro studies combined with our X-ray structures of activated and zymogen MASP-2 we built a structural model showing that the substrate-binding site of activated MASP-2 can readily accommodate the activation site segment of zymogen MASP-2 due to the high-level structural plasticity of the MASP-2 zymogen (14). We also argued that the structural models are fully compatible with zymogen MASP-2 autoactivation. Nevertheless structural modeling has its own limitations and it seems that although such autocatalytic process is not structurally hindered, it proceeds at a very low, biologically irrelevant rate. This very low rate might be a reflection of an evolutionary past when MASP-2 zymogen autoactivation might have been biologically relevant.

Another important conclusion is that zymogen MASP-1 is a potent activator of both MASP1 and MASP-2 proenzymes. This suggests that once the PRM binds to its target molecular pattern the very first catalytic step is carried out by zymogen MASP-1, which means that proenzyme MASP-1 is the principal conductor of the entire activation process. Once zymogen MASP-1 
becomes activated it gains an overwhelming capacity to activate both proenzyme MASP-1 and MASP-2.

The activation mechanism or PRM-MASP complexes

Although by combining many different types of experiments we could finally construct a coherent model of consecutive enzymatic events, we note that it is still largely unknown how these steps are orchestrated topologically into the context of the various PRM complexes. Using small-angle X-ray scattering and electron microscopy measurements, it was shown recently that in the MBL-MASP-1 complex (that contains a dimer of zymogen MASP-1 molecules) the serine protease domains of the MASP-1 protomers are protruded from the cone defined by the collagenous stalks of MBL (33). It suggests that the catalytic region of MASP-1 is always accessible for the substrates even in the fluid phase. This is in sharp contrast to the previous models of the zymogen C1 complex where the catalytic domains of the $\mathrm{C} 1 \mathrm{r}$ and $\mathrm{C} 1 \mathrm{~s}$ serine proteases are embedded inside the cone (34-36). After ligand binding by $\mathrm{C} 1 \mathrm{q}$, the zymogen activation steps occur intramolecularly and, as a result of a significant conformational change, the catalytic domains of the C1s molecules emerge at the outer surface of the C1q scaffold. The situation in the case of the lectin pathway activation could be quite different. Degn et al. (37) showed that clustering of the MBL/ficolin-MASP complexes on a proper activation surface results in the crossactivation of the zymogen proteases in an intercomplex manner. According to this model the conformational changes followed by the ligand binding of PRMs do not play a role in the activation process, what is really important is the correct juxtaposition of the activation complexes on the surface. Although the evidences supporting for both models are convincing, it seems quite intriguing that the classical and the lectin pathways use quite different mechanisms for activation. Further investigations are needed to resolve this paradox.

\section{Interactions with other complement pathways}

\section{General considerations}

Since the alternative pathway serves as an amplification loop for both the classical and the lectin pathways, there is an obvious connection between the three activation pathways of complement. In this part other possible connection points will be discussed regarding the roles of MASPs and MBL in the initiation of the other two pathways. The similarity of the lectin pathway complexes with $\mathrm{C} 1$ suggests that their components might cross-act during the formation of the classical C3 convertase.

There are also many indications that the lectin pathway plays an important role in initiation of the alternative pathway, however the mechanistic details are largely unknown. Nevertheless MASP-3 has emerged recently as the activator of pro-FD, which provides an established link 
between the two pathways and also a well defined function for MASP-3. Other potential but still controversial links between the two pathways are also discussed.

\section{Lectin and classical pathway analogies and potential cross-binding}

The complement activating capacity of MBL has been originally described through the interaction of $\mathrm{MBL}$ and the $\mathrm{C}_{1} \mathrm{r}_{2} \mathrm{~S}_{2}$ tetramer of the classical pathway $(1,38)$. Especially the higher oligomeric forms of MBL (the pentameric and hexameric variants) were efficient in activating the $\mathrm{C}_{1} \mathrm{r}_{2} \mathrm{~S}_{2}$ tetramer (2). These early experiments showed that the frame of the MBL molecule is compatible with the proteases of the classical pathway. A thorough study by Phillip and colleagues (35) provided quantitative data on cross-binding interactions between C1q and MASP dimers, and also on PRMs of the lectin pathway and the $\mathrm{C}_{1} \mathrm{r}_{2} \mathrm{~S}_{2}$ tetramer. These cross-binding interactions are generally quite strong, and they are in the range of typical protein-protein interactions $\left(\mathrm{K}_{\mathrm{d}} \sim 30\right.$ $100 \mathrm{nM})$, however the interaction strength of the proteases with the cognate PRMs are at least 10 -fold stronger. The relative affinities suggest that non-cognate complexes might exist in vivo, though likely at low proportion compared to cognate complexes. On the other hand in case of genetic deficiencies their proportion might be more significant.

It is also noteworthy that the only natural inhibitor of the classical pathway, C1 inhibitor, is also a main inhibitor of both MASP-1 and MASP-2 $(39,40)$. In this respect the regulation of the two pathways are strongly interconnected.

The $\mathrm{C}_{1} \mathrm{r}_{2} \mathrm{~S}_{2}$ tetramer is formed through the heteromeric interaction between the $\mathrm{N}$-terminal CUB1-EGF-CUB2 regions of the two enzymes. In this regard the $\mathrm{C} 1$ complex can be viewed as a hexameric $\mathrm{C} 1 \mathrm{q}$ molecule accommodating two $\mathrm{C} 1 \mathrm{r}-\mathrm{C} 1 \mathrm{~s}$ heterodimers. Analogous interactions were described for MASP-1, MASP-2 and MBL as well. MASP-1 and MASP-2 can also form heterodimers, at least in vitro (41), and co-complexes of MASP-1, MASP-2 and MBL were detected in human serum $(42,43)$. These co-complexes can either contain one MASP-1-MASP-2 heterodimer, or two homodimers of each enzyme.

In all the architecture of $\mathrm{C} 1$ and lectin pathway PRM-MASP complexes is very similar, many analogies exist, their regulation is common, and possible in vivo cross-binding of PRMs with noncognate proteases cannot be ruled out.

\section{MASP-3 activates pro-FD of the alternative pathway}

Factor $D(F D)$ catalyses the proteolytic reaction that leads to the formation of the alternative pathway C3 convertase. FD has a very restricted substrate specificity, it cleaves factor B (FB) in complex with $\mathrm{C} 3 \mathrm{~b}$, but not free FB (44). This role of FD is essential for the initiation of the alternative pathway. For a long time factor $D$ has been regarded by most complementologists to exist only in the active form in the circulation (45), and it was thought that FD is activated at the site of the synthesis possibly intracellularly (46). Although it is true that FD is predominantly 
present in the active form in normal blood, the previous picture has become questionable when Takahashi and colleagues (47) detected only the proenzyme form of FD (pro-FD) in the serum of KO mice lacking both MASP-1 and MASP-3, and consequently no alternative pathway activity was observed. Their original explanation was that pro-FD is activated by MASP-1, because recombinant MASP-1 (rMASP-1) was capable of doing so in vitro using purified proteins. However, adding rMASP-1 to the KO serum did not result in pro-FD activation (47). Later it was proposed that, in addition to MASP-1, MASP-3 can also activate pro-FD, and even the proenzyme form of MASP-3 was suggested to be able to convert pro-FD to FD (48). Meanwhile, others have detected a functional alternative pathway in the serum of a $3 \mathrm{MC}$ (49) patient lacking both MASP-1 and MASP3 concluding that neither enzyme is required for alternative pathway function (29).

In order to resolve this obvious discrepancy we have decided to use a different approach. We aimed to follow the conversion of pro-FD to FD directly in various types of blood samples (i.e. plasma and serum) in the presence or absence of specific inhibitors against candidate activators.

At first we determined the cleavage rates of pro-FD by all three MASPs testing both the activated and proenzyme forms, and by thrombin. All active MASPs and thrombin were found to be efficient pro-FD activators in vitro, on the other hand the proenzymes had no significant activity (50). These results imply that pro-FD can probably be activated by various active proteases with P1 Arg specificity in vitro, and the real question is which one of these enzymes (or another enzyme) is in the active form when pro-FD activation takes place. In order to follow pro-FD activation in complex samples such as serum or plasma pro-FD was labeled with Cy3 fluorescent dye. Because the difference in the mobility of FD and pro-FD on SDS-PAGE is very small Cy3-pro-FD was purified to ensure that only single labeled pro-FD was used in these experiments. When Cy3-pro-FD was added to citrated normal human plasma it was converted to Cy3-FD showing that proteolytic activity capable of activating pro-FD was present in this sample (50). Since in citrated plasma neither the complement system nor the coagulation system is expected to be activated we concluded that a pro-FD activator is present in normal human blood under "resting" conditions. This is consistent with the previous observation that in normal blood the predominant form of FD is the active enzyme (45).

When our previously developed MASP-1 specific inhibitor, SGMI-1, and the MASP-2 specific inhibitor, SGMI-2, was added at concentrations (100 nM) capable of completely inhibiting the corresponding enzymes, pro-FD activation was not significantly altered in plasma, suggesting that neither of these enzymes is a direct pro-FD activator. Surprisingly, at high micromolar SGMI-2 concentration pro-FD activation was slowed-down significantly. Incidentally SGMI-2 happens to be a very poor MASP-3 inhibitor $\left(\mathrm{K}_{\mathrm{i}} \sim 5 \mu \mathrm{M}\right)(28)$, hence the effect was consistent with MASP-3 being the candidate for pro-FD activation. This suspicion was confirmed when the active recombinant catalytic fragments of MASP-1, MASP-2, and MASP-3 were added separately to plasma along with labeled pro-FD, and only MASP-3 accelerated the conversion of pro-FD to FD (50). These results strongly implicated a role of MASP-3 in pro-FD activation, however the involvement of other proteases could not be ruled out. 
Therefore we recently developed an inhibitor specific for activated MASP-3. Our latest results using this MASP-3 specific inhibitor (Dobó, Szakács, Oroszlán, Kortvely, Kiss, Boros, Szász, Závodszky, Gál, and Pál unpublished results) have unambiguously proven that active MASP-3 is the exclusive and direct activator of pro-FD in resting human blood (Fig. 4). This novel inhibitor completely blocked the activation of labeled pro-FD in citrated plasma, EDTA plasma, and even in hirudin plasma. Low level activation was still detectable in serum in the presence of the MASP-3 specific inhibitor suggesting that other enzymes (e.g. thrombin) can serve as pro-FD activators, but only during ongoing coagulation. This observation can explain why alternative pathway activity can be seen in MASP-3 deficient serum in certain cases $(29,51)$. In MASP-3 sufficient blood, however, there is no need for other enzymes, since active MASP-3 activates pro-FD efficiently in the absence of the activation of coagulation cascade. Our results also imply that a significant portion of MASP-3 has to be in the active form even in resting blood.

\section{Potential roles of MASPs and MBL in alternative pathway activation}

In the following part other implicated roles of MASPs and MBL in the activation of the alternative pathway are discussed, however, these roles are either debated or the mechanism is not yet established and require further studies to prove their in vivo relevance. MASP-1 was shown early after its discovery to directly cleave C3 (52), however the cleavage rate was rather low (7). MASP-1 actually exhibited a 30-fold higher activity toward $\mathrm{C} 3\left(\mathrm{NH}_{3}\right)$, the inactivated form of $\mathrm{C} 3$ resembling $\mathrm{C} 3\left(\mathrm{H}_{2} \mathrm{O}\right)$ than toward intact $\mathrm{C} 3$ (7). In this respect it is questionable whether MASP-1 is capable of directly activate the alternative pathway causing C3b deposition. MASP-2 was shown to exhibit similar activity toward C3, however, considering the 20-fold lower concentration of MASP-2 compared to MASP-1, this activity seems to be even less relevant (7).

MASP-2 was shown to be involved in the pathomechanism of renal ischemia reperfusion injury in a mouse model. MASP-2 deficiency reduced C3 deposition in a C4 independent fashion compared to wild-type mice (53). This indicates that MASP-2 might be involved somehow in the activation of the alternative pathway, and the mechanism is different from the established lectin pathway activation route. Similarly, MBL dependent activation of the alternative pathway was published. This activation was independent of C2, C4 (54), moreover it was shown to be independent of MASP-2. The mechanistic details of this activation are largely unknown, though MASP-1 may possibly be involved in the process.

The active form of MASP-3, as described above, activates pro-FD. In addition to its pro-FD cleaving activity MASP-3 was also implicated in direct FB activation (48). One might wonder though which protease(s) activate(s) MASP-3, especially in resting blood. MASP-1 has been shown to be able to activate MASP-3 (30) in vitro, and the possibility that MASP-2 has similar activity is also viable. In this regard MASP-1 can contribute, although indirectly, to the alternative pathway as postulated by Takahashi et al (47). 
Taken together the lectin pathway of complement is probably intricately intertwined with the alternative pathway, but our knowledge about the mechanistic details, with the exception of pro-FD activation by MASP-3, is very limited.

\title{
Interaction between the different cascade systems in the blood
}

\author{
Common origin of proteolytic cascades
}

The complement and the coagulation systems are closely related in terms of evolution and function. The horseshoe crab, which is regarded as a "living fossil" of 500 million-years, has a single proteolytic cascade system that fulfills both the roles of coagulation and immune defense at the same time (55). Later in evolution, seemingly distinct cascade systems diverged from the ancient one with more specialized functions. Although, for practical and didactical reasons we distinguish between complement, coagulation and other cascades in vertebrates, we must keep in mind that these systems communicate with each other in many ways. In many pathophysiological situations (e.g. in case of injury) both the complement and the coagulation systems are activated at the same time, and the two systems augment each-other's efficacy through cross activation (56). This could be a beneficial phenomenon when restoring the homeostasis of the body. On the other hand, the synergistic effect of the proteolytic cascades can result in the development of uncontrolled inflammatory conditions that could be detrimental if not treated. In the case of the cardiovascular diseases the simultaneous activation of the complement and the coagulation systems may contribute to the development and worsening of atherosclerosis and atherothrombosis (57). The cross talk between the blood-borne proteolytic cascades is a subject of several reviews $(58,59)$. In this chapter we concentrate only on the role of the lectin pathway, especially that of MASPs, in the coagulation and in the bradykinin generation.

The role of MASP-1 and MASP-2 in coagulation

MASP-1 can be viewed as an atypical complement protease, since it has much more relaxed substrate specificity compared to other members of the C1r/C1s/MASPs family of serine proteases. While C1r, C1s and MASP-2 have only a few substrates typically among the components of the complement system, MASP-1 has many substrates including non-complement proteins. The first non-complement substrates identified for MASP-1 were fibrinogen and Factor XIII (plasma transglutaminase) (60-62). Analysis of the proteolytic activity of MASP-1 by using synthetic oligopeptide substrate libraries demonstrated that MASP-1 is a thrombin-like enzyme: at the P1 site it shows extreme Arg selectivity and it cleaves typical thrombin substrates such as VPR-AMC at high rate $(7,63)$. Using purified proteins, MASP-1 cleaves fibrinogen and liberates fibrinopeptide $B$, a chemotactic factor for neutrophils (61). Unlike thrombin, however, it does not generate 
fibrinopeptide A which indicates that MASP-1 cleaves the $\alpha$-chain of fibrinogen differently. It was shown that MASP-1 cleaves and activates Factor XIII similarly to thrombin although with lower efficiency. An interesting observation is that MASP-1 cleaves the Val34 (P4 residue) polymorphic variant of Factor XIII faster than the Leu 34 variant, while thrombin shows the opposite preference (64). Since the Leu34 variant shows protective effect in cardiovascular diseases it is speculated that the MASP-1 mediated cleavage of the Val34 variant might be disadvantageous and may contribute to the higher incidence of thrombotic events associated with the Val34 variant. MASP-1 also directly activates thrombin-activatable fibrinolysis inhibitor (TAFI) (64). TAFI is a carboxypeptidase (carboxypeptidase B2) which prevents fibrinolysis by removing C-terminal lysines from partially digested fibrin clot thereby preventing binding of tissue type plasminogen activator. Intriguingly it also inactivates anaphylatoxins ( $\mathrm{C} 3 \mathrm{a}, \mathrm{C} 5 \mathrm{a}$ ) more efficiently than plasma carboxypeptidase $\mathrm{N}(65,66)$.

The role of MASP-1 in coagulation was further studied in plasma and in whole blood (67). Addition of MASP-1 to plasma or whole blood induced and promoted clot formation by a thrombin-dependent manner. It was also demonstrated that MASP-1 cleaves and activates prothrombin. Although the cleavage sites preferred by MASP-1 (R155, R271, R393) do not exactly match that of Factor Xa (R320, R393) and thrombin (autolytic cleavage at R155 and R284), MASP-1 generate thrombin species that are active in coagulation (68). These results indicate that the clot formation ability of MASP-1 in a physiological setting is a consequence of its proteolytic action on prothrombin rather than the direct cleavage of fibrinogen. An interesting observation is that MASP-1 has a clear influence on the final structure of the clot. The proteolytic activity of MASP-1 in the plasma resulted in the formation of clot having a less dense structure but with thicker fibrin fibers (64). A less dense structure usually means shorter lysis time. Surprisingly this clot is more resistant to fibrinolysis than the clot formed without addition of MASP-1. A possible explanation could be that MASP-1 activity promotes incorporation of complement components (e.g. C3b) into the fibrin mesh. The TAFI activating ability of MASP-1 can also diminish the efficacy of the fibrinolysis.

Another study showed that MASP-2 is also capable of activating prothrombin, moreover in an in vitro experiment, binding of MBL-MASP-2 complexes to bacterial surface (S. aureus) elicited fibrin deposition when incubated with purified prothrombin and fibrinogen (69). The thrombin generating potential of MASP-2 is quite low (about 4-5\% the rate observed with purified Factor $\mathrm{Xa}$ ) and this value would be even lower in a pathophysiological situation where Factor $X$ a is present in the prothrombinase complex together with Factor $V a$ on negatively charged phospholipid membranes. It is hard to estimate the contribution of MASP-2 to the prothrombin activation in a pathophysiological situation, but given that the concentration of MASP-2 in the plasma is much lower than that of MASP-1 and the activation of MASP-2 is MASP-1 dependent, we can conclude that the procoagulant activity of the lectin pathway is driven mainly by MASP-1. This assumption is supported by two in vivo observations: 1.) MASP-1 knock-out mice show prolonged bleeding time upon tail tip excision (70), and 2.) MASP-1 knock-out mice have significantly decreased ferric 
chloride-induced occlusive arterial thrombogenesis (71). Moreover, MAp44 (alias MAP-1), a natural truncated N-terminal fragment of MASP-1 which is capable of displacing MASPs in the MBL-MASPs complexes also showed protective effects in the ferric chloride coagulation (thrombus formation) model (72).

The procoagulant activity of the lectin pathway may reflect an ancient form of innate immune defense, which is not widely explored in mammals. Limited, localized coagulation, fibrin polymer formation may limit the spread of invading microorganisms within the body (73). Fibrin deposited on the surface of the pathogens may immobilize them, cause their aggregation, or at least serve as adhesion points for phagocytes that are recruited by the released fibrinopeptides.

\section{Common regulators}

In the light of the above facts it is not surprising that the regulation of the two cascade systems is also closely connected. The major inhibitors for the complement and coagulation proteases are serpins in the blood. C1 inhibitor is regarded as the major inhibitor of the classical and the lectin pathways, since it inactivates $\mathrm{C} 1 \mathrm{r}, \mathrm{C} 1 \mathrm{~s}, \mathrm{MASP}-1$ and MASP-2 by trapping them into a covalent enzyme-inhibitor complex (74). C1 inhibitor also has anti-coagulant effect by inhibiting Factor Xla and Factor XIla. We have shown recently, that antithrombin, a serpin responsible for the regulation of thrombin, is a very effective lectin pathway inhibitor (40). In fact, in the presence of heparin antithrombin is a better inhibitor of MASP-1 than C1 inhibitor (22). Very recently two independent publications corroborated the role of antithrombin in lectin pathway regulation detecting MASP-1-antithrombin complexes in the blood of patients having various diseases (e.g. $\operatorname{HAE}, \operatorname{SLE}$, polytrauma) $(75,76)$. The latter publication also demonstrates that blood clotting activates the lectin pathway (76). Both activated platelets and fibrin clots triggered the activation of MASP-1 and MASP-2. If this is the case, then the two systems, the coagulation and the lectin pathways, activate each-other reciprocally mounting a powerful response by means of a positive amplification circle. If we want to prevent harmful activation of the cascade systems (e.g. in the case of thrombosis) we should target both pathways simultaneously, just like the natural, endogenous inhibitors ( $\mathrm{C} 1$ inhibitor, antithrombin) do.

\section{MASP-1 and the kinin generating system}

Bradykinin is an important inflammatory mediator peptide: it causes vasodilatation, increases vascular permeability and elicits production of second-generation mediators (77). It is generated from high-molecular-weight kininogen as a result of the activation of the kallikrein-kinin cascade (78). Uncontrolled release of bradykinin during hereditary angioedema (HAE) leads to recurrent tissue swelling attacks. HAE is characterized by functional C1 inhibitor deficiency. Besides the complement and the coagulation systems $\mathrm{C} 1$ inhibitor also regulates the kallikreinkinin cascade. Plasma kallikrein, the executive enzyme of the cascade, that is responsible for 
bradykinin generation, is also inhibited by C1 inhibitor. Using a proteomic approach we discovered that high-molecular-weight kininogen is a potential natural substrate of MASP-1 (79). Recombinant MASP-1 and purified natural MBL-MASPs complexes were able to cleave highmolecular-weight kininogen and liberate bradykinin. Although the efficiency of this process is low compared to the efficiency of the plasma kallikrein mediated cleavage, it could be important for two reasons. First, during infection, when the lectin pathway activates locally, the bradykinin production could contribute to local inflammation of the tissues, which is a part of the innate immune response. Second, since C1 inhibitor is a natural inhibitor of plasma kallikrein and MASP1 , the reduced functional $\mathrm{C} 1$ inhibitor concentration in the HAE patients results in the increased activation of MASP-1 yielding an excess bradykinin production. Taken into account the high autoactivation potential of MASP-1, it is also possible that MASP-1 contributes to the elevated baseline level of bradykinin observed in the blood of the HAE patients (80). Indeed, a recent publication reported that that there is a significant amount of circulating MASP-1-C1 inhibitor complex in the resting blood of healthy individuals (75). This means that MASP-1 is being continuously (auto)activated in the plasma at a slow rate (similarly to the "tick over" of C3), but the active MASP-1 is present only for a limited period of time, since it is inactivated by C1 inhibitor (and possibly by antithrombin). In the meantime, however, active MASP-1 can digest kininogen and probably other potential physiologically important substrates. In the HAE patients the uncontrolled activation of MASP-1 can lead to excess bradykinin generation that could contribute to the initiation and/or the maintenance of the edematous attacks.

\section{The promiscuity of MASP-1}

As we have seen, MASP-1 can interact with numerous substrates and inhibitors. This is quite atypical among the complement serine proteases which usually have very restricted substrate specificity. The crystal structure of MASP-1 explains the unique features of this serine protease (22). The substrate binding cleft of MASP-1 is relatively large and open capable of accommodating different substrate molecules, while the same region of C1r, C1s and MASP-2 is restricted by bulky side chains. The catalytic domain of MASP-1 resembles that of trypsin rather than other complement proteases. Nevertheless, there are two structural features that restrict the activity and specificity of MASP-1. Similarly to Factor D, the crucial Asp residue on the bottom of the substrate binding pocket is engaged with an Arg side chain through a salt bridge. The unusually long Loop B (60-loop), as in the case of thrombin, may constrict the substrate specificity.

Taken together, MASP-1 is an atypical complement protease, which can cleave and activate many proteins of the coagulation system maintaining and ancient link between blood clotting and innate immune response (Fig. 5).

\section{The role of MASPs in cell activation}




\section{Interaction between humoral and cellular immunity}

Similarly to the extensive interaction of plasma cascade systems described above, subsets of immune system are also intertwined and synergistic. To understand the function of such subsystems (e.g. T cell response, complement system, NK cells) didactic separation was needed and amenable, however, when the immune response occurs in vivo, none of these systems works alone. We have recognized the interaction between innate- and adaptive immunity (e.g. the activation of complement classical pathway), and the synergism of humoral and cellular immunity (e.g. the antibody dependent cell-mediated cytotoxicity) long ago. Initially, complement system was considered as a cascade of soluble proteins, which has a single ultimate goal: to lyse the invading microbial cells. In vitro and in vivo experiments, as well as the exploration of deficiencies of different complement factors, have taught us that complement system is a fine-tuned regulator of immunity, it can be activated via several distinct molecular patterns and specific receptors (i.e. antibodies), and it differentially responds to these stimuli by opsonization, chemotaxis initiation, cell activation and lysis. Why is it important to have multiple routes of crosstalk between complement system and cellular immunity? To overwhelm invading microbes or clear away the cellular debris of damaged tissues, kinetics is substantially important. The earlier the immune response is turned on the higher chance of the body has to win this battle. Moreover, pathogens evolved a wide variety of escape mechanisms to avoid the immune system. Crosstalk between the parts of immune system decreases the effectiveness of such microbial tricks.

\section{MASP-1 activates endothelial cells through PAR cleavage}

MASP-1 is associated with lectin pathway pattern recognition receptors (MBL, CL-K1, CL-L1 and ficolins) and is readily autoactivated upon the pathogen/danger associated molecular patterns are recognized by the lectin pathway receptors. This occurs within minutes and is under the control of only a few serine protease inhibitors, mainly $\mathrm{C} 1$ inhibitor and antithrombin. We showed that MASP-1 (similarly to thrombin) can cleave protease activated receptors (PARs) on the surface of endothelial cells, which results in the pro-inflammatory activation of endothelial cells (81). Activation of p38 MAPK, PCREB, NFKB and JNK pathways along with Ca-mobilization led to cytokine production and altered adhesion molecule pattern in a HUVEC model (82, 83). Elevated IL-8 secretion and E-selectin expression resulted in increased neutrophil chemotaxis and adhesion forces toward endothelial cells. The endothelial cell stimulating capability of MASP-1 is attributed only to fully active MASP-1 molecules. Neither zymogen form nor serine-to-alanine mutated inactive form of MASP-1 was able to activate HUVECs (84). These findings support our hypothesis that microbial infection or tissue damage is necessary to trigger MASP-1 induced, endothelial cell driven neutrophil activation (Fig. 6). The direct effect of MASP-1 on neutrophil granulocytes or other subsets of leukocytes has not been studied yet. It has long been known that anaphylatoxins ( $\mathrm{C} 3 \mathrm{a}, \mathrm{C} 5 \mathrm{a}$ ) possess similar neutrophil chemotactic and activating effects as the products of MASP-1 
induced endothelial cells. Therefore, the question emerges: why do we need such overlapping functions of two subsequent factors of the complement system? The neutrophil attracting and activating effects of MASP-1 are triggered solely by the lectin pathway activity, and are regulated by $\mathrm{C} 1$ inhibitor and at endothelial cell level but not by other complement regulatory proteins. In contrast, $\mathrm{C} 3 \mathrm{a}$ and $\mathrm{C} 5 \mathrm{a}$, as central products of all three complement pathways, are controlled by several complement regulators (e.g. C4BP, factor H, DAF, MCP, factor I) but not by endothelial gene expression. Therefore, the synergism of complement system and cellular immunity (especially neutrophil granulocytes in this context) by MASP-1 and anaphylatoxins completes each other's function providing fine-tuned immune regulation (Fig. 6). Liver is the predominant organ for the synthesis of plasma proteins, including most of the complement factors and other proteins participating in the acute phase response. Hepatic stellate cells, the hepatic equivalent of pericytes, can also be activated by MASP-1 ( 85 ), which results in a fibrinogenic myofibroblast phenotype. This reaction also requires the active form of MASP-1: zymogen form is similarly inactive as in the endothelial cell model. Although the physiological role of quiescent hepatic stellate cells are largely unknown, they may interact with other hepatic cell types, such as hepatocytes, liver sinusoidal endothelial cells and Kupffer cells (86), and may be involved in tolerogenic antigen presentation of the liver (87).

\section{Possible role of MASP-2 and MASP-3 in cell activation}

We know much less about the role of MASP-2 beyond lectin pathway activation. In contrast to MASP-1, MASP-2 has a stringent substrate binding specificity. In fact, it has no other known substrates than $\mathrm{C} 2, \mathrm{C} 4$ and possibly prothrombin, therefore it was not unexpected that we could not detect any effects of MASP-2 on HUVECs (81). However, Asgari et al. found that MASP-2 has a critical role in the renal ischemia/reperfusion injury even in the absence of C4 (53). It suggests the presence of other substrate(s) of MASP-2 than C4 and C2, since C4 is necessary to the complement activation through the lectin pathway, whereas C2 cleavage can also be accomplished by MASP-1. In this model, MASP-2 deposition could be detected on the peritubular surface, which raises the question whether MASP-2 is able to interact with cells directly. Moreover, van der Pol et al. also described similar detrimental role of $\mathrm{MBL}$ in renal ischemia/reperfusion injury (88). Although they found that $\mathrm{C} 3$ or downstream complement activation is not responsible for the action of MBL, the possible role of MASP-2 (and the other MASPs) was not excluded. If MASP-2 has indeed a direct effect on various cell types, the question is still open, which receptor(s) it can use, since MASP-2 has no PAR cleaving activity.

The substrate specificity, the possible natural inhibitors and the exact physiological role of MASP-3 is still largely unknown. Besides its factor D activating ability, published recently (50), MASP-3 is involved in several processes in ontogenesis, demonstrated by the bone-, neuronal- and sexual- malformations associated with its mutation in $3 M C$ syndrome $(49,89,90)$. Although MASP- 
3 may cleave soluble hormone-like proteins to exert its action (91), we could not exclude the direct cell activating effects of MASP-3 either.

Apart from their enzymatic activity, all MASPs can influence the interaction of MBL or Lficolin with CR1, since their binding site to the collagen stalks of lectin pathway receptors are overlapping (92). However, it is still not known, whether this in vitro competition is relevant in vivo, and what the possible consequences of the reaction are.

In summary, MASPs (and especially MASP-1) are not only the keystone enzymes of the complement lectin pathway and a participant of other plasma cascade systems, but they can activate different cell types implicating an intimate crosstalk between humoral immune system and other cellular systems of the body through MASPs, which leads to a more effective and more complex immune response.

\section{Conclusion and perspectives}

As we have seen, the MASPs play multiples roles in the initiation of the innate immune response. MASP-1 and MASP-2 are the indispensable enzymes responsible for lectin pathway activation, while the proteolytic activity of MASP-3 represents a key linkage between the lectin and the alternative pathway. MASPs also contribute to physiological processes outside the complement activation. MASP-1 and MASP-2 may be involved in other proteolytic cascades, such as the coagulation and the kinin generation pathways. MASP-1 has also been shown to directly activate different cells connecting the humoral and cellular immunity. These findings demonstrate that the complement system is a part of a lager proteolytic network and MASPs have multiple interaction partners (substrates, inhibitors, PRMs) in this network. In this respect exciting discoveries are expected in the future. The role of MASP-3 in the embryonic development is also an intriguing question. Although MASP-3 seems to be a protease with restricted substrate specificity and low activity, its deficiency leads to the development of $3 \mathrm{MC}$ syndrome. The only established known MASP-3 substrate is FD, however FD deficiency does not cause 3MC syndrome (but that of CL-K1 does). We can conclude that MASP-3 (especially CL-K1-MASP-3 complex) has at least one unidentified substrate that is important in the ontogenesis. The uncontrolled activity of MASPs may also contribute to the development of various disease conditions. HAE, ischemiareperfusion injury, atypical haemolytic uraemic syndrome, age-related macular degeneration, rheumatoid arthritis are only a few examples of diseases where inhibition of the proteolytic activity of MASPs may have therapeutic benefit. Development of MASP inhibitors (small molecules, recombinant proteins, inhibitory antibodies) are underway and may be tested soon in the clinical practice (93). 


\section{Acknowledgments}

This study was supported by the Hungarian Scientific Research Fund (OTKA) grants NK100834, NK100769, K100684, K115623 and K108642 as well as by the National Development Agency Grant KMOP-4.2.1/B-10-2011 and the MedInProt program of the Hungarian Academy of Sciences.

The authors have no conflicts of interest to disclose. 


\section{References}

1. Ikeda K, Sannoh T, Kawasaki N, Kawasaki T, Yamashina I. Serum lectin with known structure activates complement through the classical pathway. J Biol Chem 1987;262:7451-7454.

2. Lu JH, Thiel S, Wiedemann H, Timpl R, Reid KB. Binding of the pentamer/hexamer forms of mannan-binding protein to zymosan activates the proenzyme C1r2C1s2 complex, of the classical pathway of complement, without involvement of C1q. J Immunol 1990;144:22872294.

3. Pillemer L, Blum L, Lepow IH, Ross OA, Todd EW, Wardlaw AC. The properdin system and immunity. I. Demonstration and isolation of a new serum protein, properdin, and its role in immune phenomena. Science 1954;120:279-285.

4. Degn SE, Thiel S. Humoral pattern recognition and the complement system. Scand J Immunol 2013;78:181-193.

5. Matsushita M, Fujita T. Activation of the classical complement pathway by mannose-binding protein in association with a novel C1s-like serine protease. J Exp Med 1992;176:1497-1502.

6. Thiel S, et al. A second serine protease associated with mannan-binding lectin that activates complement. Nature 1997;386:506-510.

7. Ambrus G, et al. Natural substrates and inhibitors of mannan-binding lectin-associated serine protease-1 and -2: a study on recombinant catalytic fragments. J Immunol 2003;170: 1374-1382.

8. Vorup-Jensen $\mathrm{T}$, et al. Distinct pathways of mannan-binding lectin (MBL)- and C1-complex autoactivation revealed by reconstitution of $\mathrm{MBL}$ with recombinant $\mathrm{MBL}$-associated serine protease-2. J Immunol 2000;165:2093-2100.

9. Dahl MR, et al. MASP-3 and its association with distinct complexes of the mannan-binding lectin complement activation pathway. Immunity 2001;15:127-135.

10. Zundel $S$, et al. Characterization of recombinant mannan-binding lectin-associated serine protease (MASP)-3 suggests an activation mechanism different from that of MASP-1 and MASP-2. J. Immunol. 2004;172:4342-4350

11. Stover $\mathrm{CM}$, et al. Two constituents of the initiation complex of the mannan-binding lectin activation pathway of complement are encoded by a single structural gene. J Immunol 1999;162:3481-3490.

12. Degn SE, Hansen AG, Steffensen R, Jacobsen C, Jensenius JC, Thiel S. MAp44, a human protein associated with pattern recognition molecules of the complement system and regulating the lectin pathway of complement activation. J Immunol 2009;183:7371-7378.

13. Schwaeble WJ, et al. Targeting of mannan-binding lectin-associated serine protease-2 confers protection from myocardial and gastrointestinal ischemia/reperfusion injury. Proc Natl Acad Sci U S A 2011;108:7523-7528. 
14. Gál P, Harmat V, Kocsis A, Bián T, Barna L, Ambrus G, Végh B, Balczer J, Sim RB, Náray-Szabó G, Závodszky P. A true autoactivating enzyme. Structural insight into mannose-binding lectinassociated serine protease-2 activations. J Biol Chem 2005;280:33435-33444.

15. Schwaeble WJ, Dahl MR, Thiel S, Stover C, Jensenius JC. The mannan-binding lectinassociated serine proteases (MASPs) and MAp19: four components of the lectin pathway activation complex encoded by two genes. Immunobiol 2002;205:455-466

16. Rossi V, Cseh S, Bally I, Thielens NM, Jensenius JC, Arlaud GJ. Substrate specificities of recombinant mannan-binding lectin-associated serine proteases-1 and -2. J Biol Chem 2001;276:40880-40887.

17. Chen $\mathrm{CB}$, Wallis R. Two mechanisms for mannose-binding protein modulation of the activity of its associated serine proteases. J Biol Chem 2004;279:26058-26065.

18. Matsushita M, Thiel S, Jensenius JC, Terai I, Fujita T. Proteolytic activities of two types of mannose-binding lectin-associated serine protease. J Immunol 2000;165: 2637-2642.

19. Møller-Kristensen M, Thiel S, Sjöholm A, Matsushita M, Jensenius J C. Cooperation between MASP-1 and MASP-2 in the generation of C3 convertase through the MBL pathway. Int Immunol 2007;19:141-149.

20. Takahashi $\mathrm{M}$, et al. Mannose-binding lectin (MBL)-Associated serine protease (MASP)-1 contributes to activation of the lectin complement pathway. J Immunol 2008;180:61326138.

21. Harmat V, Gál P, Kardos J, Szilágyi K, Ambrus G, Végh B, Náray-Szabó G, Závodszky P. The structure of MBL-associated serine protease-2 reveals that identical substrate specificities of C1s and MASP-2 are realized through different sets of enzyme-substrate interactions. J Mol Biol 2004;342:1533-1546.

22. Dobó J, Harmat V, Beinrohr L, Sebestyén E, Závodszky P, Gál P. MASP-1, a promiscuous complement protease: structure of its catalytic region reveals the basis of its broad specificity. J Immunol 2009;183:1207-1214.

23. Schechter I, Berger A. On the size of the active site in proteases. I. Papain. Biochem Biophys Res Commun 1967;27:156-162.

24. Kocsis A, Kékesi KA, Szász R, Végh BM, Balczer J, Dobó J, Závodszky P, Gál P, Pál G. Selective inhibition of the lectin pathway of complement with phage display selected peptides against mannose-binding lectin-associated serine protease (MASP)-1 and -2: significant contribution of MASP-1 to lectin pathway activation. J Immunol 2010;185:4169-4178.

25. Seelen MA, et al. Functional analysis of the classical, alternative, and MBL pathways of the complement system: standardization and validation of a simple ELISA. J Immunol Methods 2005;296:187-198.

26. Petersen SV, Thiel S, Jensen L, Steffensen R, Jensenius JC. An assay for the mannan-binding lectin pathway of complement activation. J Immunol Methods 2001;257:107-116.

27. Héja D, Harmat V, Fodor K, Wilmanns M, Dobó J, Kékesi KA, Závodszky P, Gál P, Pál G. Monospecific inhibitors show that both mannan-binding lectin-associated serine protease-1 
(MASP-1) and -2 Are essential for lectin pathway activation and reveal structural plasticity of MASP-2. J Biol Chem 2012;287:20290-20300.

28. Héja D, Kocsis A, Dobó J, Szilágyi K, Szász R, Závodszky P, Pál G, Gál P. Revised mechanism of complement lectin-pathway activation revealing the role of serine protease MASP-1 as the exclusive activator of MASP-2. Proc Natl Acad Sci U S A 2012;109:10498-10503.

29. Degn SE, Jensen L, Hansen AG, Duman D, Tekin M, Jensenius JC, Thiel S. Mannan-binding lectin-associated serine protease (MASP)-1 is crucial for lectin pathway activation in human serum, whereas neither MASP-1 nor MASP-3 is required for alternative pathway function. J Immunol 2012;189:3957-3969.

30. Megyeri M, Harmat V, Major B, Végh Á, Balczer J, Héja D, Szilágyi K, Datz D, Pál G, Závodszky $P$, Gál P, Dobó J. Quantitative characterization of the activation steps of mannan-binding lectin (MBL)-associated serine proteases (MASPs) points to the central role of MASP-1 in the initiation of the complement lectin pathway. J Biol Chem 2013;288:8922-8934.

31. Møller-Kristensen $\mathrm{M}$, et al. Levels of mannan-binding lectin-associated serine protease-2 in healthy individuals. J Immunol Methods 2003;282:159-167.

32. Thiel $\mathrm{S}$, et al. Mannan-binding lectin (MBL)-associated serine protease-1 (MASP-1), a serine protease associated with humoral pattern-recognition molecules: normal and acute-phase levels in serum and stoichiometry of lectin pathway components. Clin Exp Immunol 2012;169:38-48.

33. Kjaer TR, et al. Structural insights into the initiating complex of the lectin pathway of complement activation. Structure 2015;23:342-351.

34. Bally I, Rossi V, Lunardi T, Thielens NM, Gaboriaud C, Arlaud GJ. Identification of the C1qbinding Sites of Human C1r and C1s: a refined three-dimensional model of the C1 complex of complement. J Biol Chem 2009;284:19340-19348.

35. Phillips $A E$, et al. Analogous interactions in initiating complexes of the classical and lectin pathways of complement. J Immunol 2009;182:7708-7717.

36. Venkatraman Girija $\mathrm{U}$, et al. Structural basis of the $\mathrm{C} 1 \mathrm{q} / \mathrm{C} 1 \mathrm{~s}$ interaction and its central role in assembly of the C1 complex of complement activation. Proc Natl Acad Sci U S A 2013;110:13916-13920.

37. Degn $\mathrm{SE}$, et al. Complement activation by ligand-driven juxtaposition of discrete pattern recognition complexes. Proc Natl Acad Sci U S A 2014;111:13445-13450.

38. Ohta M, Okada M, Yamashina I, Kawasaki T. The mechanism of carbohydrate-mediated complement activation by the serum mannan-binding protein. J Biol Chem 1990;265:19801984.

39. Petersen SV, Thiel S, Jensen L, Vorup-Jensen T, Koch C, Jensenius JC. Control of the classical and the MBL pathway of complement activation. Mol Immunol 2000;37:803-811.

40. Paréj K, Dobó J, Závodszky P, Gál P. The control of the complement lectin pathway activation revisited: both $\mathrm{C} 1$-inhibitor and antithrombin are likely physiological inhibitors, while $\alpha 2-$ macroglobulin is not. Mol Immunol 2013;54:415-422. 
41. Paréj K, Hermann A, Donáth N, Závodszky P, Gál P, Dobó J. Dissociation and re-association studies on the interaction domains of mannan-binding lectin (MBL)-associated serine proteases, MASP-1 and MASP-2, provide evidence for heterodimer formation. Mol Immunol 2014;59:1-9.

42. Degn SE, Jensen L, Olszowski T, Jensenius JC, Thiel S. Co-complexes of MASP-1 and MASP-2 associated with the soluble pattern-recognition molecules drive lectin pathway activation in a manner inhibitable by MAp44. J Immunol 2013;191:1334-1345.

43. Rosbjerg A, Munthe-Fog L, Garred P, Skjoedt MO. Heterocomplex formation between $\mathrm{MBL} /$ ficolin/CL-11-associated serine protease-1 and -3 and MBL/ficolin/CL-11-associated protein-1. J Immunol 2014;192:4352-4360.

44. Forneris F, Ricklin D, Wu J, Tzekou A, Wallace RS, Lambris JD, Gros P. Structures of C3b in complex with factors $B$ and $D$ give insight into complement convertase formation. Science 2010;330:1816-1820.

45. Lesavre PH, Müller-Eberhard HJ. Mechanism of action of factor $D$ of the alternative complement pathway. J Exp Med 1978;148:1498-1509.

46. Volanakis JE, Narayana SV. Complement factor D, a novel serine protease. Protein Sci 1996;5:553-564.

47. Takahashi $M$, et al. Essential role of mannose-binding lectin-associated serine protease-1 in activation of the complement factor D. J Exp Med 2010;207:29-37.

48. Iwaki D, Kanno K, Takahashi M, Endo Y, Matsushita M, Fujita T. The role of mannose-binding lectin-associated serine protease-3 in activation of the alternative complement pathway. J Immunol 2011;187:3751-3758.

49. Sirmaci A, et al. MASP1 mutations in patients with facial, umbilical, coccygeal, and auditory findings of Carnevale, Malpuech, OSA, and Michels syndromes. Am J Hum Genet 2010;87:679-686.

50. Oroszlán G, et al. MASP-1 and MASP-2 Do Not Activate Pro-Factor D in Resting Human Blood, whereas MASP-3 Is a Potential Activator: Kinetic Analysis Involving Specific MASP-1 and MASP-2 Inhibitors. J Immunol 2016;196:857-865.

51. Ruseva MM, Takahashi M, Fujita T, Pickering MC. C3 dysregulation due to factor $\mathrm{H}$ deficiency is mannan-binding lectin-associated serine proteases (MASP)-1 and MASP-3 independent in vivo. Clin Exp Immunol 2014;176:84-92.

52. Matsushita M, Fujita T. Cleavage of the third component of complement (C3) by mannosebinding protein-associated serine protease (MASP) with subsequent complement activation. Immunobiology 1995;194:443-448.

53. Asgari E, et al. Mannan-binding lectin-associated serine protease 2 is critical for the development of renal ischemia reperfusion injury and mediates tissue injury in the absence of complement C4. FASEB J 2014;28:3996-4003. 
54. Selander B, Mårtensson $U$, Weintraub A, Holmström E, Matsushita $M$, Thiel S, Jensenius JC, Truedsson L, Sjöholm AG. Mannan-binding lectin activates C3 and the alternative complement pathway without involvement of C2. J Clin Invest 2006;116:1425-1434.

55. Iwanaga S. The molecular basis of innate immunity in the horseshoe crab. Curr Opin Immunol 2002;14:87-95.

56. Amara $U$, et al. Molecular intercommunication between the complement and coagulation systems. J Immunol 2010;185:5628-5636.

57. Speidl WS, Kastl SP, Huber K, Wojta J. Complement in atherosclerosis: friend or foe? J Thromb Haemost 2011;9:428-440.

58. Amara $U$, et al. Interaction between the coagulation and complement system. Adv Exp Med Biol 2008;632:71-79.

59. Conway EM. Reincarnation of ancient links between coagulation and complement. J Thromb Haemost 2015;Suppl 1:S121-132.

60. Hajela $\mathrm{K}$, et al. The biological functions of MBL-associated serine proteases (MASPs). Immunobiology 2002;205:467-475.

61. Krarup A, Gulla KC, Gál P, Hajela K, Sim RB. The action of MBL-associated serine protease 1 (MASP1) on factor XIII and fibrinogen. Biochim Biophys Acta 2008;1784:1294-1300.

62. Gulla KC, et al. Activation of mannan-binding lectin-associated serine proteases leads to generation of a fibrin clot. Immunology 2010;129:482-495.

63. Presanis JS, Hajela K, Ambrus G, Gál P, Sim RB. Differential substrate and inhibitor profiles for human MASP-1 and MASP-2. Mol Immunol 2004;40:921-929.

64. Hess K, Ajjan R, Phoenix F, Dobó J, Gál P, Schroeder V. Effects of MASP-1 of the complement system on activation of coagulation factors and plasma clot formation. PLoS One 2012;7:e35690.

65. Myles $\mathrm{T}$, et al. Thrombin activatable fibrinolysis inhibitor, a potential regulator of vascular inflammation. J Biol Chem 2003;278:51059-51067.

66. Nishimura T, Myles T, Piliponsky AM, Kao PN, Berry GJ, Leung LL. Thrombin-activatable procarboxypeptidase B regulates activated complement C5a in vivo. Blood 2007;109:19921997.

67. Jenny L, Dobó J, Gál P, Schroeder V. MASP-1 of the complement system promotes clotting via prothrombin activation. Mol Immunol 2015a;65:398-405.

68. Jenny L, Dobó J, Gál P, Schroeder V. MASP-1 Induced Clotting - The First Model of Prothrombin Activation by MASP-1. PLoS One 2015b;10:e0144633.

69. Krarup A, Wallis R, Presanis JS, Gál P, Sim RB. Simultaneous activation of complement and coagulation by MBL-associated serine protease 2. PLoS One 2007;2:e623.

70. Takahashi $\mathrm{K}$, et al. Mannose-binding lectin and its associated proteases (MASPs) mediate coagulation and its deficiency is a risk factor in developing complications from infection, including disseminated intravascular coagulation. Immunobiology 2011;216:96-102. 
71. La Bonte $L R$, et al. Mannose-binding lectin-associated serine protease-1 is a significant contributor to coagulation in a murine model of occlusive thrombosis. J Immunol 2012;188:885-891.

72. Pavlov VI, Skjoedt MO, Siow Tan Y, Rosbjerg A, Garred P, Stahl GL. Endogenous and natural complement inhibitor attenuates myocardial injury and arterial thrombogenesis. Circulation. 2012;126:2227-2235.

73. Dunn DL, Simmons RL. Fibrin in peritonitis. III. The mechanism of bacterial trapping by polymerizing fibrin. Surgery. 1982;92:513-519.

74. Davis AE 3rd, Lu F, Mejia P. C1 inhibitor, a multi-functional serine protease inhibitor. Thromb Haemost 2010;104:886-893.

75. Hansen CB, et al. The Levels of the Lectin Pathway Serine Protease MASP-1 and Its Complex Formation with C1 Inhibitor Are Linked to the Severity of Hereditary Angioedema. J Immunol 2015;195:3596-3604.

76. Kozarcanin $\mathrm{H}$, et al. The lectin complement pathway serine proteases (MASPs) represent a possible crossroad between the coagulation and complement systems in thromboinflammation. J Thromb Haemost 2016;14:531-545.

77. Oehmcke S, Herwald H. Contact system activation in severe infectious diseases. J Mol Med 2010;88:121-126.

78. Kaplan AP, Joseph $\mathrm{K}$. The bradykinin-forming cascade and its role in hereditary angioedema. Ann Allergy Asthma Immunol 2010;104:193-204.

79. Dobó J, et al. Cleavage of kininogen and subsequent bradykinin release by the complement component: mannose-binding lectin-associated serine protease (MASP)-1. PLoS One. 2011;6:e20036.

80. Joseph K, Tuscano TB, Kaplan AP. Studies of the mechanisms of bradykinin generation in hereditary angioedema plasma. Ann Allergy Asthma Immunol 2008;101:279-286.

81. Megyeri $\mathrm{M}$, et al. Complement protease MASP-1 activates human endothelial cells: PAR4 activation is a link between complement and endothelial function. J Immunol 2009;183:3409-3416.

82. Jani PK, et al. MASP-1 Induces a Unique Cytokine Pattern in Endothelial Cells: A Novel Link between Complement System and Neutrophil Granulocytes. PLoS One 2014;9:e87104.

83. Jani PK, et al. Complement MASP-1 enhances adhesion between endothelial cells and neutrophils by up-regulating E-selectin expression. Mol Immunol 2016;75:38-47.

84. Megyeri $M$, et al. Serum MASP-1 in complex with MBL activates endothelial cells. Mol Immunol 2014;59:39-45.

85. Saeed $A$, et al. Mannan binding lectin-associated serine protease 1 is induced by hepatitis $C$ virus infection and activates human hepatic stellate cells. Clin Exp Immunol 2013;174:26573.

86. Kmieć Z. Cooperation of liver cells in health and disease. Adv Anat Embryol Cell Biol 2001;161:III-XIII, 1-151. 
87. Dunham RM, et al. Hepatic stellate cells preferentially induce Foxp3+ regulatory $\mathrm{T}$ cells by production of retinoic acid. J Immunol 2013;190:2009-2016.

88. van der Pol $\mathrm{P}$, et al. Mannan-binding lectin mediates renal ischemia/reperfusion injury independent of complement activation. Am J Transplant 2012;12:877-887.

89. Rooryck $C$, et al. Mutations in lectin complement pathway genes COLEC11 and MASP1 cause 3MC syndrome. Nat Genet 2011;43:197-203.

90. Degn SE, Jensenius JC, Thiel S. Disease-causing mutations in genes of the complement system. Am J Hum Genet. 2011;88:689-705.

91. Cortesio CL, Jiang W. Mannan-binding lectin-associated serine protease 3 cleaves synthetic peptides and insulin-like growth factor-binding protein 5. Arch Biochem Biophys 2006;449:164-170.

92. Jacquet $M$, et al. Deciphering complement receptor type 1 interactions with recognition proteins of the lectin complement pathway. J Immunol 2013;190:3721-3731.

93. Morgan BP, Harris CL. Complement, a target for therapy in inflammatory and degenerative diseases. Nat Rev Drug Discov 2015;14:857-877. 


\section{Figure legends}

Fig. 1. Domain structure of MASPs and related proteins. MASP-1, MASP-3 and MAp44 are the products of the MASP1 gene. MASP-1 and MASP-3 differ only in the SP domain, while MAp44 is a shortened protein with 17 unique C-terminal amino acids following the CCP1 domain. MASP-2 and MAp19 are the products of the MASP2 gene. MAp19 is a shortened protein with 4 unique amino acids at the C-terminus following the EGF domain. Shared domains with the same sequence are depicted with identical colors.

Fig. 2. Mechanism of the major arm of the complement lectin pathway. When PRM-MASP complexes bind to activator surfaces MASP-1 autoactivates first, then it activates MASP-2. MASP-2 cleaves $\mathrm{C} 4$ and both enzymes can activate $\mathrm{C} 2$, resulting in the formation of the classical C3 convertase $\mathrm{C} 4 \mathrm{bC2}$. Red arrows represent proteolytic cleavage pointing from the enzyme to the substrate. Gray arrows designate conversion, while gray curved lines indicate a non-enzymatic signal.

Fig. 3. Zymogen MASP-1 is the initiator of lectin pathway activation. Zymogen and active MASP-1 and MASP-2 activities were measured on themselves (autoactivation) and on each-other (crossactivation) using recombinant catalytic fragments. Non-activatable mutant variants served as models for the zymogen state, while the wild-type enzymes were used to measure the activities of active state. The active-site Ser to Ala mutants, which are completely inactive, were used as substrates. The $\mathrm{k}_{\mathrm{obs}} /[\mathrm{E}]_{\mathrm{T}}$ values were calculated, which are as good estimates for the catalytic efficiencies $\left(k_{c a t} / K_{M}\right)$. Please note the logarithmic scale on the $y$ axis! MASP-1 is the main driver during the entire process, however, in the initial zymogen phase only MASP-1 has significant activity, hence the whole activation process is dependent on the inherent activity of zymogen MASP-1.

Fig. 4. Fundamental link between the lectin and the alternative complement pathways. MASP-3, component of the lectin pathway, was found to be the major activator of pro-FD in general, and the exclusive activator of pro-FD in resting human blood. Only active MASP-3 can activate pro-FD. Since FD is an essential initiator of the alternative pathway, the presence of active MASP-3 is required for the normal functioning of the alternative pathway. Red arrows represent proteolytic cleavage pointing from the enzyme to the substrate, while gray arrows indicate conversion. 
Fig. 5. The promiscuity of MASP-1. In addition to its complement activating capacity, MASP-1 was also shown to be able to boost coagulation, and contribute to a more powerful inflammatory reaction through activation of endothelial cells and bradykinin release. The inhibitors of MASP-1 are shared between the complement system and coagulation.

Fig. 6. The lectin pathway crosstalk hypothesis. Microbial (especially bacterial and fungal) infections and damaged self structures provide danger signals (PAMPs and DAMPs, respectively) to the immune system. They are perceived by pattern recognition molecules, such as complement lectin pathway receptors: MBL, ficolins and collectins. Upon this recognition, MASP-1

autoactivates. At this point the response branches, active MASP-1 can cleave MASP-2, which carry on the complement activation providing chemotactic factors (anaphylatoxins) opsonins and membrane attack complexes to hamper the impaired homeostatic conditions. Simultaneously, MASP-1 activates endothelial cells to produce pro-inflammatory cytokines and adhesion molecules, which together facilitate neutrophil granulocyte chemotaxis to the injured tissues and adhesion to the endothelium. Neutrophils, as being the most prominent cell type of the immune system against bacteria and fungi, activated by the MASP-1-induced endothelial cells, can be further activated by anaphylatoxins and utilize opsonins to clear microbes and cellular debris with high efficiency. This novel cooperation between neutrophils and complement lectin pathway is orchestrated by MASP-1. 
Fig. 1

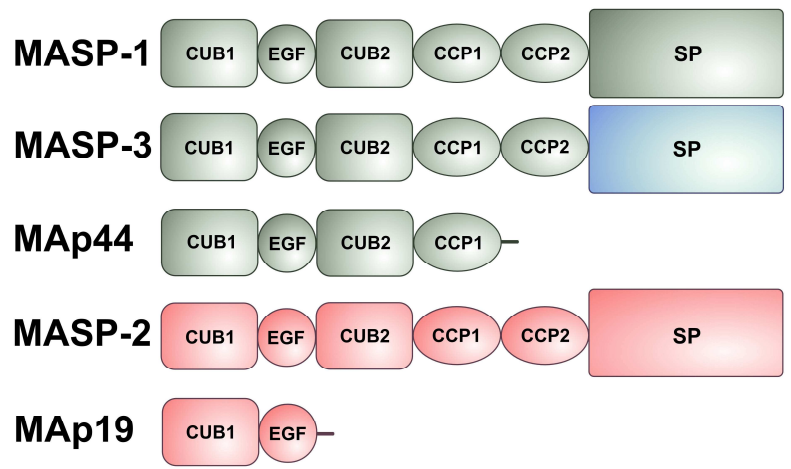

Fig. 3

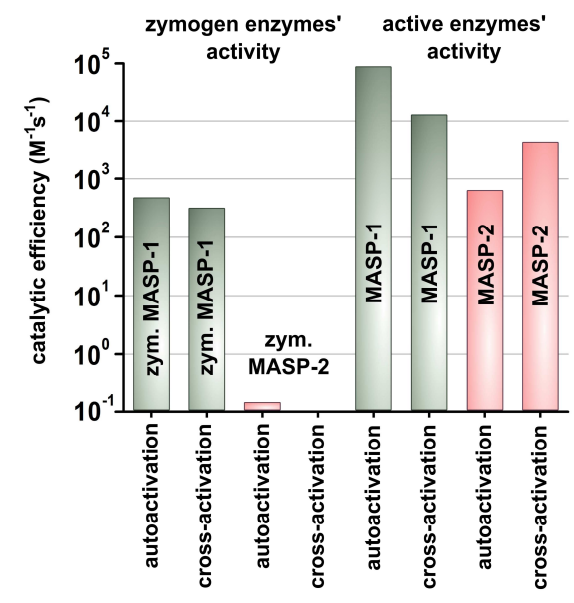

Fig. 5

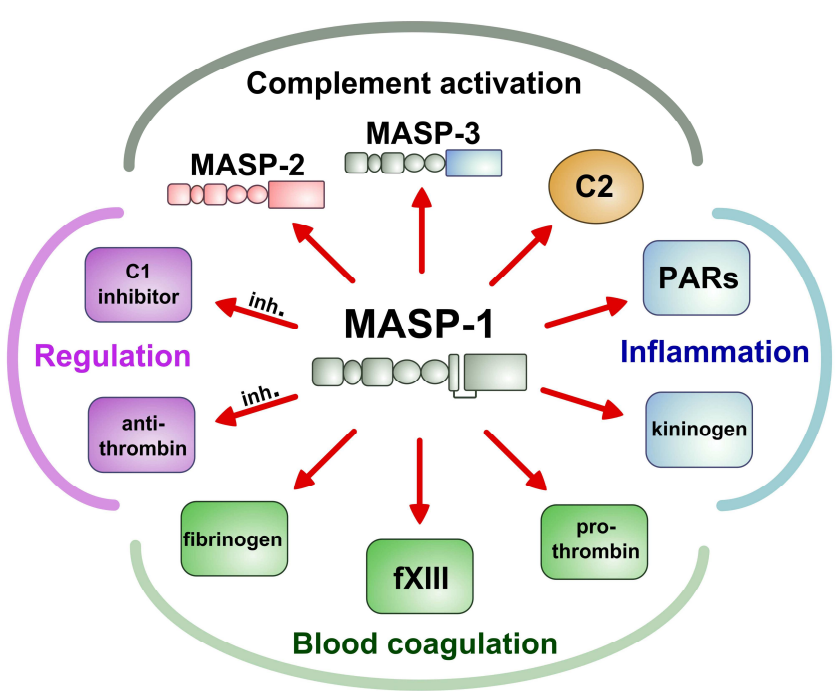

Fig. 2

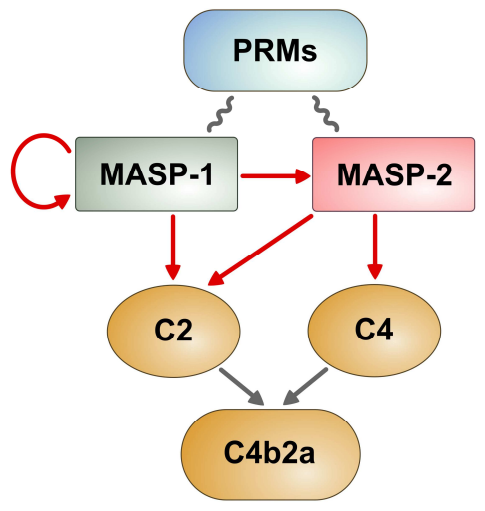

Fig. 4

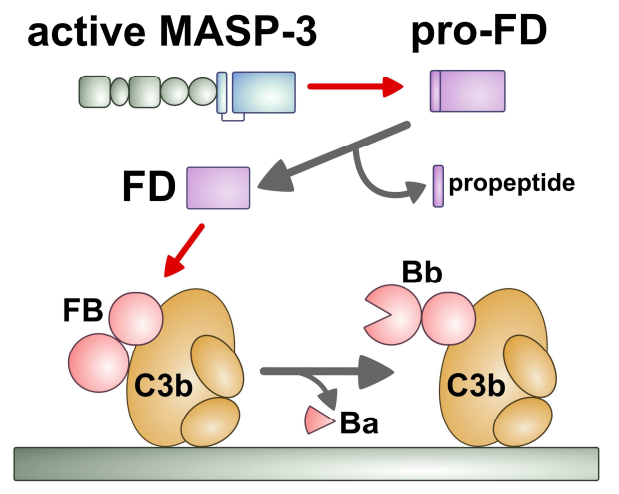

Fig. 6

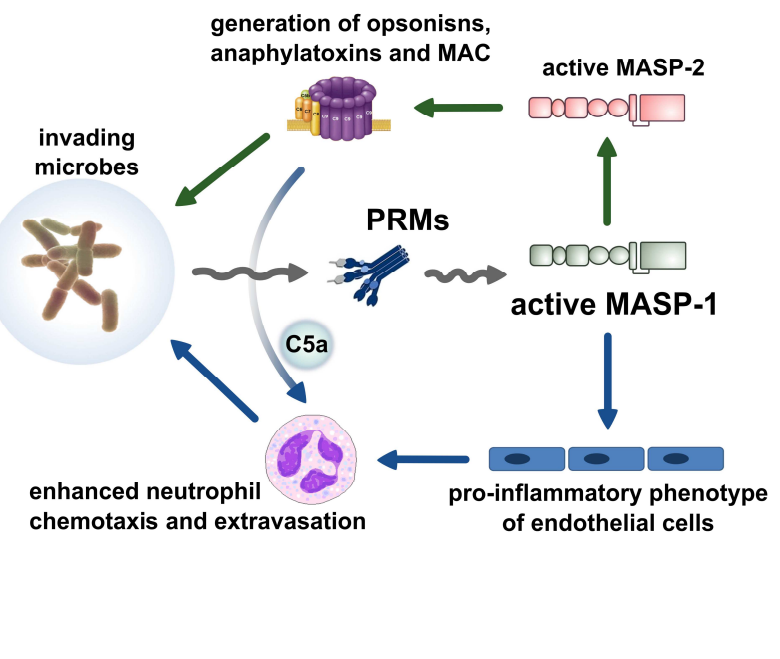

